\title{
Secreted fungal sulfhydryl oxidases: sequence analysis and characterisation of a representative flavin-dependent enzyme from Aspergillus oryzae
}

\author{
Greta Faccio ${ }^{*}$, Kristiina Kruus, Johanna Buchert, Markku Saloheimo
}

\begin{abstract}
Background: Sulfhydryl oxidases are flavin-dependent enzymes that catalyse the formation of de novo disulfide bonds from free thiol groups, with the reduction of molecular oxygen to hydrogen peroxide. Sulfhydryl oxidases have been investigated in the food industry to remove the burnt flavour of ultraheat-treated milk and are currently studied as potential crosslinking enzymes, aiming at strengthening wheat dough and improving the overall bread quality.

Results: In the present study, potential sulfhydryl oxidases were identified in the publicly available fungal genome sequences and their sequence characteristics were studied. A representative sulfhydryl oxidase from Aspergillus oryzae, AoSOX1, was expressed in the fungus Trichoderma reesei. AoSOX1 was produced in relatively good yields and was purified and biochemically characterised. The enzyme catalysed the oxidation of thiol-containing compounds like glutathione, D/L-cysteine, beta-mercaptoethanol and DTT. The enzyme had a melting temperature of $57^{\circ} \mathrm{C}$, a pH optimum of 7.5 and its enzymatic activity was completely inhibited in the presence of $1 \mathrm{mM} \mathrm{ZnSO}$.

Conclusions: Eighteen potentially secreted sulfhydryl oxidases were detected in the publicly available fungal genomes analysed and a novel proline-tryptophan dipeptide in the characteristic motif CXXC, where $X$ is any amino acid, was found. A representative protein, AoSOX1 from A. oryzae, was produced in T. reesei in an active form and had the characteristics of sulfhydryl oxidases. Further testing of the activity on thiol groups within larger peptides and on protein level will be needed to assess the application potential of this enzyme.
\end{abstract}

\section{Background}

Disulfide bonds are essential for the stability and function of intracellular and secreted proteins. The subject of this work are sulfhydryl oxidases, SOX, and in particular glutathione oxidases (E.C. 1.8.3.3), enzymes catalysing the formation of de novo disulfide bonds between thiol groups with the subsequent reduction of oxygen to hydrogen peroxide (equation 1).

$$
2 \mathrm{R}-\mathrm{SH}+\mathrm{O}_{2} \rightarrow \mathrm{R}-\mathrm{S}-\mathrm{S}-\mathrm{R}+\mathrm{H}_{2} \mathrm{O}_{2}
$$

The name sulfhydryl oxidase is sometimes also referred to thiol oxidases (EC 1.8.3.2), enzymes that also oxidise thiol groups using oxygen as electron acceptor but reducing it to water. Thiol oxidases have been

\footnotetext{
* Correspondence: greta.faccio@vtt.fi

VIT Technical Research Centre of Finland PO Box 1000, 02044 Espoo, Finland
}

isolated from fungi, e.g. Mycothecium [1], Piricularia and Polyporus [2].

The first secreted fungal enzyme containing FAD and able to oxidise glutathione and several sulfhydryl compounds was reported in 1982 from Penicillium sp. K-65 . This enzyme had negligible activity on cysteines in proteins. Furthermore, it was not effective on the reactivation of reduced RNase A [3]. In 1987, a secreted sulfhydryl oxidase active on protein associated thiol groups was isolated in Aspergillus niger culture filtrates [4]. The enzyme was found to be homodimeric, and each subunit was binding tightly but non-covalently a FAD molecule. $A$. niger sulfhydryl oxidase was active on glutathione and in a lesser extent on homocysteine, DTT, cysteine, a g-glu-cys dipeptide characterised by a carboxylamide bond [5] and its presence increased the rate of reactivation of reduced ribonuclease A [4]. The sulfhydryl oxidase from A. niger and Penicillium have a different 
evolutionary origin than the well-characterised intracellular sulfhydryl oxidases, of the Erv family, and are thought to be more related to thioredoxin reductases and pyridine nucleotide flavin disulfide oxidoreductases $[3,4,6]$. Their physiological role is however still unclear. Metallo-sulfhydryl oxidases containing iron [7] or copper [8] have also been reported.

The action of sulfhydryl oxidases on small thiol-containing compounds and the production of hydrogen peroxide, similarly to the well-known glucose oxidase [9], make sulfhydryl oxidase very attractive for the food industry. Sulfhydryl oxidases can be a valid alternative to the use of chemical additives, such as potassium bromate or ascorbic acid, for the improvement of the strength and handling properties of wheat dough in the baking industry.

The aim of this work was to analyze the putative secreted sulfhydryl oxidases in the publicly available fungal genomes and to produce and biochemically characterise one of the identified enzymes, i.e. AoSOX1 from Aspergillus oryzae.

\section{Results and Discussion}

\section{Analysis of secreted fungal sulfhydryl oxidases}

The search for secreted proteins carrying a predicted disulphide oxidoreductases domain of class II and, in particular, FAD-dependent ones (see Materials and method section) retrieved among 398 proteins 48 with a signal sequence, no ER retention signal and no putative transmembrane segments, and thus are highly likely to be secreted (Table 1). The only characterized protein found among them is the sulfhydryl oxidase from $A$. niger (AnSOX, [NCBI:CAK40401]) [4]. Numerous retrieved proteins have been found in the Aspergillus spp. and Neosartoria fischeri, a close relative of the Aspergilli. Alignment of the sequences identified allowed the selection of 18 proteins possessing the CXXC motif characteristic of thiol:disulfide oxidoreductases like sulfhydryl oxidases (Figure 1). Three main deletions can be identified from the alignment in Figure 1, e.g. protein [Swiss-Prot: Q2H2X8] lacks residues in position 57-83 and 302-318, and protein TRIRE0077288 (http://genome.jgi-psf.org/ Trire2/Trire2.home.html) in position 190-211 (residues numbered according to the alignment).

Sequence features typical of FAD dependent pyridine nucleotide disulphide oxidoreductases (IPR013027) were reflected in three regions. Firstly, a conserved motif characteristic of the Rossmann fold (V/I)(V/I) GXGXXGXXXA/L, where $\mathrm{X}$ is any residue, is found in the N-terminal region of the sequences (residues 38-49 in the alignment in Figure 1) suggesting that the proteins bind to a nucleotide cofactor such as FAD or $\mathrm{NAD}(\mathrm{P})$, with a $\beta \alpha \beta$-fold and possibly function as oxidoreductases [10]. The second conserved region is located in the middle of the protein sequence and contains the two conserved cysteine residues of the CXXC motif (residues 177-180 in Figure 1) that is characteristic of the thiol:disulfide oxidoreductases, including sulfhydryl oxidases and, in general, oxidoreductases of the thioredoxin fold family [11]. Many conserved residues are found in this region and the following pattern can be identified RKHHL(A/G)TGXXDX 6 GXX(E/D)XY(G/ A)XGXYYCXXC(D/H)GYE ( $\mathrm{X}$ is any amino acid, $\mathrm{H}$ a hydrophobic amino acid and $\mathrm{Y}$ an aromatic residue). Here is located the ATG motif common to FAD and NADPH-binding domains and reported to be located at the end of the fourth $\beta$-strand interacting with the cofactor [12].

The dipeptide comprised between the cysteine residues has been shown to affect the redox properties of proteins with a CXXC motif [13]. The proline-tryptophan dipeptide found in AoSOX1 and another eleven of the sulfhydryl oxidases studied here has never been characterized, but the $\mathrm{N}$-terminal proline is thought to positively affect the local conformation in DsbA, a protein required for disulfide bond formation in E. coli [14]. Quan and co-authors also showed a positive correlation between the presence of an aromatic residue in the $\mathrm{C}$ terminal position and a higher catalytic efficiency. The third conserved region is in the C-terminus and includes firstly, the GD motif (TXHXGHY(A/G)HGD, residues 340-350 in Figure 1) that is involved in the binding of the ribityl moiety of FAD in most flavoproteins with two-dinucleotide binding domains but not common in single FAD-binding domain proteins, e.g. absent in cholesterol oxidase and glucose oxidase, and secondly, the G-helix (AHXXG, residues 362-366 in Figure 1)[12,15]. The final stretch is rich in glutamic acid residues and is predicted to have intrinsic disorder, e.g., after residue E350 in AnSOX and after K349 in AoSOX1 [16].

An overall lower level of conservation is observed in sequence [Swiss-Prot:Q4W4QJ0] from Aspergillus fumigatus and [Swiss-Prot:Q55JR2] from C. neoformans. Four of the sequences retrieved (at the top of the alignment in Figure 1) clearly group separately and have a different dipeptide (LF) between the cysteine residues of the active site, e.g. [Swiss-Prot:A4QYP9] from Magnaporthe grisea, [Swiss-Prot:A1DN23] from N. fischeri, [Swiss-Prot:Q0CME9] from Aspergillus terreus and [Swiss-Prot:Q5MBU7] from A. fumigatus. The three last-named sequences have an unusually long predicted signal peptide, e.g. 28 residues for [Swiss-Prot:Q0CME9] and [Swiss-Prot:A1DN23], 27 residues for [Swiss-Prot: Q5MBU7].

\section{Production of AoSOX1 in Trichoderma reesei}

A representative protein, AoSOX1 [NCBI:BAE61582], with $64.7 \%$ level of identity to $A$. niger sulfhydryl oxidase 
Table 1 Secreted fungal sulfhydryl oxidases listed by organism and their key features

\begin{tabular}{|c|c|c|c|c|c|c|c|}
\hline Family & Organism & Name & Status & Length & $\begin{array}{l}\text { Signal } \\
\text { peptide } \\
\text { length }\end{array}$ & $\begin{array}{l}\text { Identity to } \\
\text { AnSOX (\%) }\end{array}$ & $\begin{array}{l}\text { CXXC } \\
\text { motif }\end{array}$ \\
\hline Chaetomiaceae & C. globosum & [Swiss-Prot:Q2H2IO]] & putative & 517 & 26 & 14.4 & - \\
\hline Chaetomiaceae & C. globosum & [Swiss-Prot:Q2H2X8] & putative & 356 & 18 & 37.9 & CPWC \\
\hline Hypocreaceae & T. reesei & TRIRE0077288 & predicted & 382 & 19 & 37.1 & CPWC \\
\hline Magnaporthaceae & M. grisea & [Swiss-Prot:A4QYP9] & putative & 325 & 20 & 24.2 & CLFC \\
\hline Magnaporthaceae & M. grisea & [Swiss-Prot:A4R670] & predicted & 398 & 19 & 40.1 & CPWC \\
\hline Onygenales & C. immitis & [Swiss-Prot:Q1DQ56] & predicted & 400 & 20 & 10.5 & - \\
\hline Phaeosphaeriaceae & P. nodorum & [Swiss-Prot:Q0ULD1] & putative & 425 & 18 & 16.5 & - \\
\hline Phaeosphaeriaceae & P. nodorum & [Swiss-Prot:Q0V1M0] & predicted & 392 & 22 & 18.0 & - \\
\hline Saccharomycetaceae & P. stipitis & [Swiss-Prot:A3LTU5] & predicted & 370 & 20 & 19.5 & - \\
\hline Saccharomycetaceae & L. elongisporua & [Swiss-Prot:A5DYY0] & uncharacterised & 393 & 32 & 14.8 & - \\
\hline Saccharomycetaceae & S. cerevisiae & [Swiss-Prot:P52923] & evidence at protein level & 378 & 21 & 17.5 & - \\
\hline Saccharomycetaceae & A. gossypii & [Swiss-Prot:Q759|7] & predicted & 2195 & 24 & 3.5 & - \\
\hline Sordariaceae & N. crassa & [Swiss-Prot:Q7SA02] & predicted & 627 & 22 & 12.7 & - \\
\hline Sordariaceae & N. crassa & [Swiss-Prot:Q7SA31] & putative & 538 & 27 & 12.3 & - \\
\hline Sordariaceae & N. crassa & [Swiss-Prot:Q7SAD4] & predicted & 589 & 19 & 12.9 & - \\
\hline Tremellaceae & C. neoformans & [Swiss-Prot:Q55JR2] & putative & 346 & 21 & 23.3 & $\mathrm{CIFC}$ \\
\hline Trichocomaceae & A. clavatus & [Swiss-Prot:A1C4Y4] & putative & 388 & 19 & 61.8 & CPWC \\
\hline Trichocomaceae & A. clavatus & [Swiss-Prot:A1CE06] & predicted & 599 & 19 & 9.4 & - \\
\hline Trichocomaceae & N. fischeri & [Swiss-Prot:A1CZW3] & putative & 386 & 19 & 62.8 & CPWC \\
\hline Trichocomaceae & N. fischeri & [Swiss-Prot:A1DD16] & predicted & 609 & 20 & 10.3 & - \\
\hline Trichocomaceae & N. fischeri & [Swiss-Prot:A1DJl6] & putative & 382 & 23 & 14.8 & - \\
\hline Trichocomaceae & N. fischeri & [Swiss-Prot:A1DKE0] & putative & 515 & 20 & 11.0 & - \\
\hline Trichocomaceae & N. fischeri & [Swiss-Prot:A1DN23] & putative & 334 & 28 & 25.8 & CLFC \\
\hline Trichocomaceae & N. fischeri & [Swiss-Prot:A1DPI7] & putative & 387 & 23 & 46.2 & CPWC \\
\hline Trichocomaceae & A. niger & [Swiss-Prot:A2QUK3] & AnSOX & 392 & 19 & 100.0 & CPWC \\
\hline Trichocomaceae & A. niger & [Swiss-Prot:A2R6C0] & predicted & 440 & 23 & 9.0 & - \\
\hline Trichocomaceae & A. terreus & [Swiss-Prot:Q0C958] & predicted & 360 & 28 & 19.4 & - \\
\hline Trichocomaceae & A. terreus & [Swiss-Prot:QOCBT8] & predicted & 381 & 21 & 14.4 & - \\
\hline Trichocomaceae & A. terreus & [Swiss-Prot:Q0CE26] & predicted & 387 & 19 & 65.4 & CPWC \\
\hline Trichocomaceae & A. terreus & [Swiss-Prot:Q0CME9] & putative & 335 & 28 & 25.8 & CLFC \\
\hline Trichocomaceae & A. terreus & [Swiss-Prot:Q0CW0] & predicted & 453 & 21 & 14.9 & - \\
\hline Trichocomaceae & A. terreus & [Swiss-Prot:QoCZE7] & putative & 519 & 48 & 10.8 & - \\
\hline Trichocomaceae & A. terreus & [Swiss-Prot:Q0D021] & putative & 388 & 18 & 55.3 & CPWC \\
\hline Trichocomaceae & A. oryzae & [Swiss-Prot:Q2TXY9] & predicted & 518 & 41 & 10.1 & - \\
\hline Trichocomaceae & A. oryzae & [Swiss-Prot:Q2U4P3] & predicted & 389 & 23 & 47.3 & CPWC \\
\hline Trichocomaceae & A. oryzae & [Swiss-Prot:Q2U5L3] & predicted & 478 & 20 & 14.0 & - \\
\hline Trichocomaceae & A. oryzae & [Swiss-Prot:Q2UA33] & AoSOX1 & 384 & 19 & 64.7 & CPWC \\
\hline Trichocomaceae & A. oryzae & [Swiss-Prot:Q2UCA6] & predicted & 422 & 17 & 9.0 & - \\
\hline Trichocomaceae & A. oryzae & [Swiss-Prot:Q2UE40] & predicted & 625 & 28 & 14.7 & - \\
\hline Trichocomaceae & A. oryzae & [Swiss-Prot:Q2UTD4] & predicted & 502 & 28 & 12.5 & - \\
\hline Trichocomaceae & A. oryzae & [Swiss-Prot:Q2UV04] & predicted & 513 & 28 & 11.8 & - \\
\hline Trichocomaceae & A. fumigatus & [Swiss-Prot:Q4WEM5] & predicted & 386 & 19 & 62.3 & CPWC \\
\hline Trichocomaceae & A. fumigatus & [Swiss-Prot:Q4WF68] & putative & 515 & 20 & 11.3 & - \\
\hline Trichocomaceae & A. fumigatus & [Swiss-Prot:Q4WFR5] & putative & 382 & 23 & 15.8 & - \\
\hline Trichocomaceae & A. fumigatus & [Swiss-Prot:Q4WLA0] & predicted & 508 & 34 & 10.1 & - \\
\hline Trichocomaceae & A. fumigatus & [Swiss-Prot:Q4WQJ0] & inferred from homology & 392 & 29 & 21.8 & CAVC \\
\hline Trichocomaceae & A. fumigatus & [Swiss-Prot:Q5MBU7] & predicted & 334 & 27 & 26.2 & CLFC \\
\hline Ustilaginaceae & U. maydis & [Swiss-Prot:Q4PCL0] & predicted & 583 & 29 & 15.0 & - \\
\hline
\end{tabular}

\footnotetext{
${ }^{1}$ T. reesei genome, http://genome.jgi-psf.org/Trire2/Trire2.home.html
} 


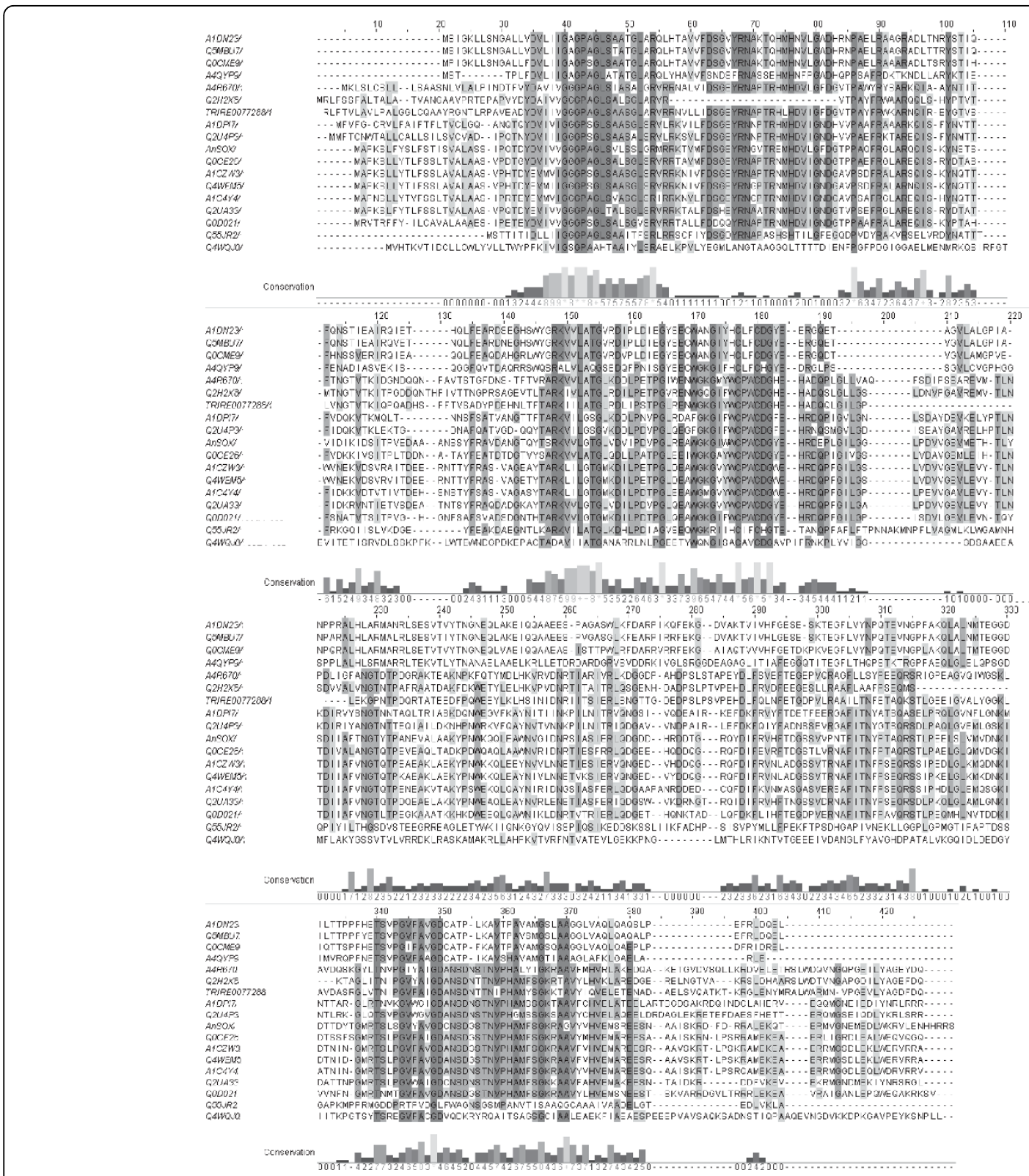

Figure 1 Sequence alignment. Sequence alignment of the candidate secreted SOX proteins containing the CXXC motif to the well-known enzyme A. niger SOX, [Swiss-Prot:A2QUK3]. Residues background intensity accords to the level of identity and the level of conservation of each residue is indicated by the histogram below and by a 0 to 5 value. The alignment was performed with ClustalW2 and visualised by JalView (http://www.jalview.org). Sequences: [Swiss-Prot:A1DN23] from N. fischeri, [Swiss-Prot:Q5MBU7] from A. fumigatus, [Swiss-Prot:Q0CME9] from A. terreus, [Swiss-Prot:A4QYP9] from M. grisea, [Swiss-Prot:A4R670] from M. grisea, [Swiss-Prot:Q2H2X8] from C. globosum, TRIRE0077288 from T. reesei, [Swiss-Prot:A1DPI7] from N. fischeri, [Swiss-Prot:Q2U4P3] from A. oryzae, AnSOX [Swiss-Prot:A2QUK3] from A. niger, [Swiss-Prot:Q0CE26] from A. terreus, [Swiss-Prot:A1CZW3] from N. fischeri, [Swiss-Prot:Q4WEM5] from A. fumigatus, [Swiss-Prot:A1C4Y4] from A. clavatus, AoSOX1 [Swiss-Prot: Q2UA33] from A. oryzae, [Swiss-Prot:Q0D021] from A. terreus, [Swiss-Prot:Q55JR2] from C. neoformans and [Swiss-Prot:Q4WQJ0] from A. fumigatus.

was chosen for expression in T. reesei and subsequent biochemical characterization. The AoSOX1 protein consists of 384 amino acids including a predicted signal sequence of 19 residues. The closest homologs to AoSOX1 are three putative thioredoxin reductases from Aspergillus flavus (99\% sequence identity, [NCBI:
EED47993]), Neosartorya fischeri (70\%, [NCBI: XP_001266180]) and A. fumigatus (68\%, [NCBI: XP_747990]). AoSOX1 gene is predicted to be interrupted by two introns.

The gene coding for AoSOX1 (1304 bp) was amplified by PCR from the genomic DNA of $A$. oryzae and cloned 
by Gateway recombination into a $T$. reesei expression vector under the control of the strong inducible $c b h 1$ promoter. A C-terminal six-histidine tag was added to the expression construct. The expression plasmid was transformed into $T$. reesei and, after screening, one clone, producing the highest activity on glutathione, was selected for enzyme production. The highest production level in shake flask was reached after 5 days of cultivation and was adequate for purification; no fermentor cultures were thus needed. The protein content of the medium at the end of the cultivation was $1.4 \mathrm{~g} / \mathrm{l}$ and AoSOX1 production level of $70 \mathrm{mg} / \mathrm{l}$ accounted for about $5 \%$ of the total secreted proteins, as estimated using the specific activity value. The production of AoSOX1 in an active form showed that the two introns were correctly spliced from the transcript. The secretion of AoSOX1 when its gene is expressed in T. reesei suggested its extracellular production also in the native strain, A. oryzae.

\section{Enzyme purification}

Attempts to purify AoSOX1 with the help of the histidine tag were unsuccessful because of the protein did not bind to copper-chelated Chelating Sepharose Fast Flow resin. Besides, Western blotting analysis of samples containing AoSOX1 with primary anti-Histag antibodies showed no recognition. Furthermore, the C-terminal peptide containing the six-histidine tag was never detected after tryptic digestion and peptide analysis. These findings suggest that possibly the histidine tag was cleaved off the recombinant enzyme by a host protease.

The AoSOX1 enzyme was purified by anion-exchange in a Hitrap DEAE FF column (elution at $166 \mathrm{mM} \mathrm{NaCl}$ concentration) and size-exclusion chromatography on a Superdex 75 HR 10/30 column (elution volume $11.3 \mathrm{ml}$ ). Results of a typical AoSOX1 purification are summarised in Table 2. AoSOX1 purification by anion exchange and size-exclusion chromatography lead to an activity yield of $68 \%$ and a purification factor of 21 . The second purification step did not increase the purity but was crucial for the removal of brown-coloured compounds probably derived from the medium used in the cultivations.

\section{Molecular characterization}

AoSOX1 in the purified form migrates in SDS PAGE in two bands at a molecular weight around 45000 and the MW determined by mass spectrometry was 43959 . The difference in molecular mass can be ascribed to N-glycosylation considering that six asparagine residues in the protein are potential $\mathrm{N}$-glycosylation sites and a net mass reduction is observed by SDS PAGE after treatment with PNGase F (Figure 2a, b). Deglycosylation resolved the two bands corresponding to AoSOX1 into a single one of apparently 40000 . This suggests that the double band is due to heterogeneity of the N-glycans.

AoSOX1 in the culture medium and in the purified form was identified by peptide mass fingerprinting in the NCBI sequence database (http://www.ncbi.nlm.nih. gov) with a maximum of $24.5 \%$ sequence coverage (Figure 2b). Peptides containing putative $\mathrm{N}$-glycosylation sites were not detected suggesting that at least some of the $\mathrm{N}$-glycosylation sites would be occupied by glycans.

\section{Biochemical characterization}

\section{Substrate specificity determination and inhibition studies}

AoSOX1 showed activity both on small thiol compounds such as cysteine and also on larger molecules such as the tripeptide glutathione (Table 3). The highest activity was registered with glutathione, similarly to the glutathione oxidase from Penicillium sp. K-6-5 [3], and the second highest with DTT. A Michaelis-Menten behaviour was observed with all the compounds tested and the highest activity was detected with glutathione as substrate. Glutathione is however an improbable physiological substrate for secreted sulfhydryl oxidases and, even though their role is still unclear, their action on cell-wall or secreted proteins, in the formation of extracellular matrix [17] or for the maturation of peptides produced non-ribosomally [18] cannot be excluded.

AoSOX1 activity was only slightly affected by the presence of the chelating compound EDTA, the salts $\mathrm{MgSO}_{4}$ and $\mathrm{MnSO}_{4}$ and the denaturing agent urea (Table 4). Similarly to glutathione oxidase from Penicillium [3], the activity of AoSOX1 was drastically reduced in presence of $\mathrm{ZnSO}_{4}$. This latter result was probably due to the interaction of the zinc ion with cysteine residues necessary for the catalytic activity of AoSOX1.

Table 2 Purification of AoSOX1 from the $T$. reesei culture medium

\begin{tabular}{|c|c|c|c|c|c|c|}
\hline & $\begin{array}{l}\text { Volume } \\
(\mathrm{ml})\end{array}$ & $\begin{array}{l}\text { Total activity } \\
\left(10^{6} \text { nkat }\right)\end{array}$ & $\begin{array}{l}\text { Total protein } \\
\text { (mg) }\end{array}$ & $\begin{array}{l}\text { Specific } \\
\text { activity } \\
\left(10^{6} \text { nkat } \mathrm{mg}^{-1}\right)\end{array}$ & $\begin{array}{l}\text { Purification } \\
\text { factor }\end{array}$ & $\begin{array}{l}\text { Activity } \\
\text { yield } \\
(\%)\end{array}$ \\
\hline Cell free culture medium ${ }^{a}$ & 60 & 311 & 324 & 0.96 & 1 & 100 \\
\hline Hitrap DEAE FF & 108 & 261 & 13 & 20.20 & 21 & 84 \\
\hline Superdex 75 HR & 8 & 210 & 11 & 19.97 & 21 & 68 \\
\hline
\end{tabular}

a The cell-free culture medium (220 ml) was concentrated and buffer exchanged.

${ }^{\mathrm{b}}$ Enzymatic activity was measured with the HVA-peroxidase coupled assay. 


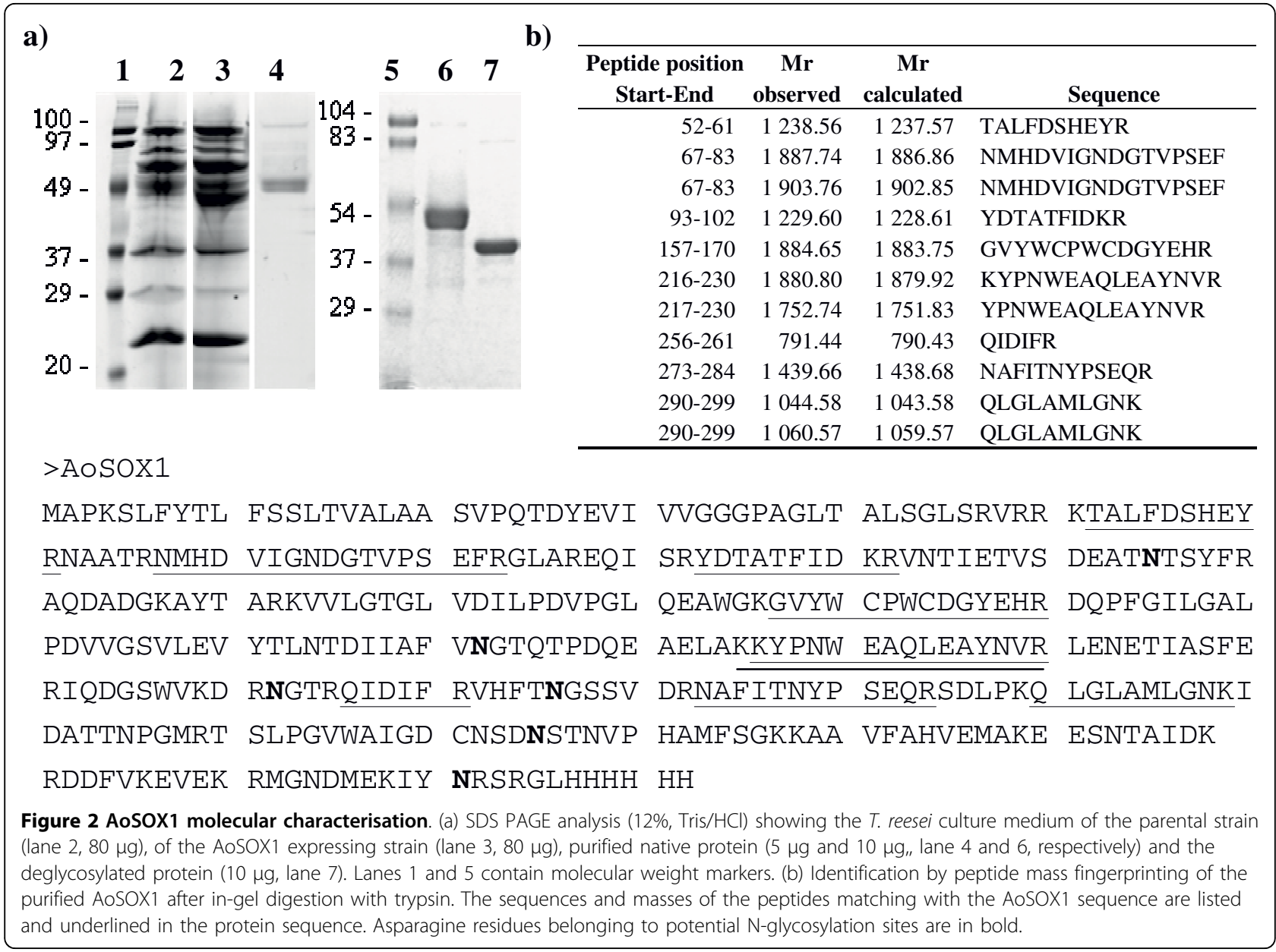

Considering the cysteine content of AoSOX1, e.g. 3 cysteines, there is a high probability that the reactive cysteines are the C177 and C180 forming the CXXC motif.

\section{$\mathrm{pH}$ and temperature behaviour}

AoSOX1 showed a significant $\mathrm{pH}$ stability retaining more than $80 \%$ of the initial activity when incubated in a pH range of 4 to 8 after 24 hours (data not shown). AoSOX1 and AnSOX showed a similar $\mathrm{pH}$ stability; the $\mathrm{pH}$ optimum value of 8 , measured for AoSOX1 (Figure 3a) was higher than the value (5.5) reported for AnSOX $[4,5]$ and similar to the ones for glutathione oxidase from Penicillium (7-7.8). AoSOX1 showed good temperature stability at 30 and $40^{\circ} \mathrm{C}$ retaining more than $70 \%$ of the initial activity after 24 hours (Figure $3 \mathrm{~b})$. The activity of AoSOX1 was reduced to $40 \%$ and $8 \%$ respectively, after one and 24 hours incubation at $50^{\circ} \mathrm{C}$. No activity was detected after 30 minutes of incubation at 60 and $70^{\circ} \mathrm{C}$ (Figure $3 \mathrm{~b}$ ).

\section{Molecular properties of AoSOX1}

The absorption spectrum of AoSOX1 showed three peaks at 275, 370 and $440 \mathrm{~nm}$ and a shoulder at 365 $\mathrm{nm}$, like for the glutathione oxidase from Penicillium [3], revealing its flavoenzymatic nature (Figure 4). The cofactor was spectrophotometrically identified to be

Table 3 Kinetic constants of AoSOX1 on five different substrates

\begin{tabular}{|c|c|c|c|c|c|}
\hline Substrate & $\begin{array}{l}\mathrm{K}_{\mathrm{m}} \\
(\mathrm{mM})\end{array}$ & $\begin{array}{l}\mathrm{V}_{\max } \\
\left(\text { nkat } \mathrm{ml}^{-1}\right)\end{array}$ & $\begin{array}{l}V_{\max } / K_{m} \\
\left(s^{-1}\right)\end{array}$ & $\begin{array}{l}\mathbf{k}_{\mathrm{cat}} \\
\left(\mathrm{s}^{-1}\right)\end{array}$ & $\begin{array}{l}\text { Efficacy number } \\
\left(\mathrm{M}^{-1} \mathrm{~s}^{-1}\right)\end{array}$ \\
\hline Glutathione & $2.78 \pm 0.56$ & $(15.3 \pm 2.89) \times 10^{7}$ & 55010 & $3.60 \times 10^{6}$ & $1.29 \times 10^{9}$ \\
\hline L-Cys & $6.11 \pm 0.13$ & $(22.2 \pm 0.57) \times 10^{5}$ & 364 & $5.23 \times 10^{4}$ & $8.56 \times 10^{6}$ \\
\hline D-Cys & $1.55 \pm 0.03$ & $(25.3 \pm 0.02) \times 10^{4}$ & 163 & $5.95 \times 10^{3}$ & $3.84 \times 10^{6}$ \\
\hline DTT [thiols] & $2.41 \pm 0.13$ & $(13.1 \pm 0.22) \times 10^{6}$ & 5451 & $3.09 \times 10^{5}$ & $1.28 \times 10^{8}$ \\
\hline$\beta$-mercaptoethanol & $9.73 \pm 0.08$ & $(21.3 \pm 0.05) \times 10^{5}$ & 219 & $5.01 \times 10^{4}$ & $5.15 \times 10^{6}$ \\
\hline
\end{tabular}


Table 4 Inhibition of AoSOX1 by various compounds

\begin{tabular}{lll}
\hline Inhibitor concentration & Residual activity ${ }^{\text {a }}$ (\%) \\
\hline EDTA & $1 \mathrm{mM}$ & $85 \pm 5$ \\
& $10 \mathrm{mM}$ & $91 \pm 7$ \\
$\mathrm{KI}$ & $1 \mathrm{mM}$ & $65 \pm 6$ \\
& $10 \mathrm{mM}$ & $48 \pm 11$ \\
$\mathrm{MgSO}_{4}$ & $1 \mathrm{mM}$ & $55 \pm 7$ \\
& $10 \mathrm{mM}$ & $80 \pm 6$ \\
$\mathrm{MnSO}_{4}$ & $1 \mathrm{mM}$ & $81 \pm 11$ \\
& $10 \mathrm{mM}$ & $94 \pm 4$ \\
$\mathrm{Na}_{2} \mathrm{SO}_{4}$ & $1 \mathrm{mM}$ & $61 \pm 10$ \\
& $10 \mathrm{mM}$ & $70 \pm 5$ \\
$\mathrm{ZnSO}_{4}$ & $1 \mathrm{mM}$ & $1 \pm 0$ \\
& $10 \mathrm{mM}$ & $3 \pm 1$ \\
$\mathrm{SDS}$ & $1 \mathrm{mM}$ & $58 \pm 10$ \\
& $10 \mathrm{mM}$ & $64 \pm 11$ \\
$\mathrm{NaCl}$ & $1 \mathrm{mM}$ & $91 \pm 12$ \\
& $10 \mathrm{mM}$ & $74 \pm 13$ \\
Urea & $1 \mathrm{mM}$ & $80 \pm 6$ \\
& $10 \mathrm{mM}$ & $79 \pm 11$ \\
\hline
\end{tabular}

${ }^{a}$ Values are expressed as means \pm standard deviations.

FAD after the cofactor was released from the enzyme by thermal and chemical denaturation (Figure 4, inset).

The secondary structure of AoSOX1 presented alphahelical elements, as evidenced by the two negative peaks at 210 and $225 \mathrm{~nm}$ in the CD spectrum (Figure 5a). Thermal denaturation of AoSOX1 revealed a melting temperature of $57^{\circ} \mathrm{C}$ (Figure $5 \mathrm{~b}$ ). The available threedimensional structures are of intracellular sulfhydryl

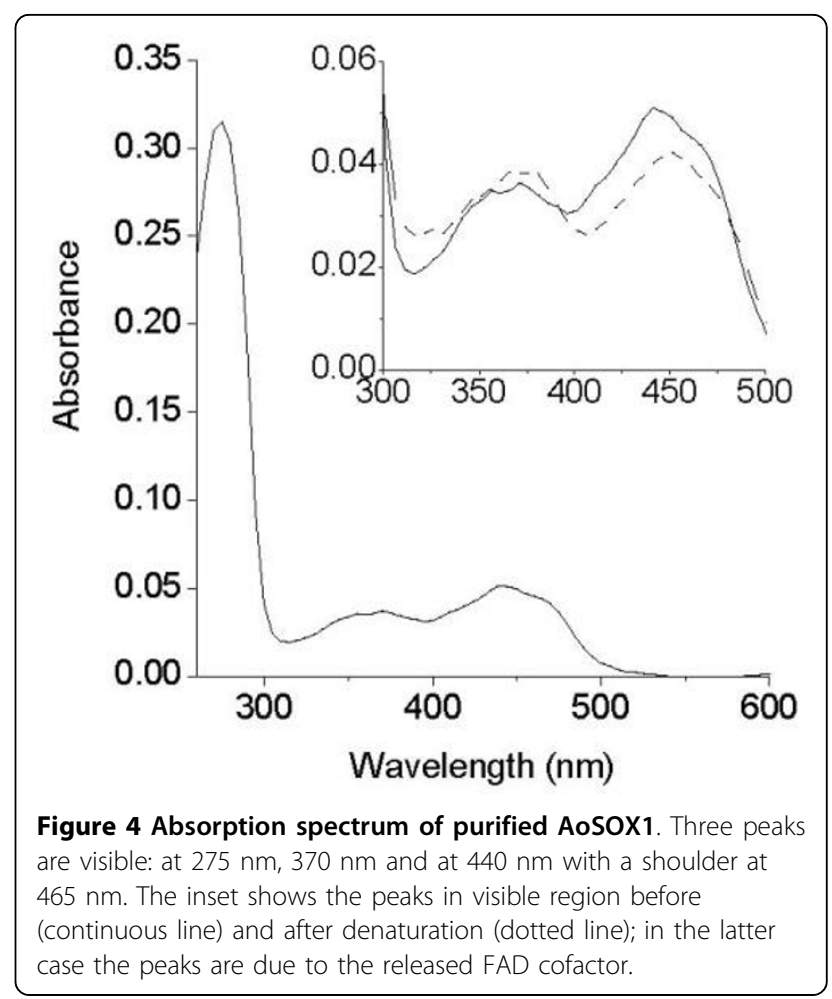

oxidases and it would be interesting to see how the structure of a secreted enzyme like AoSOX1 compares with these structures.

The stability of AoSOX1 against chaotropic denaturants, e.g. guanidinium hydrochloride, was studied by
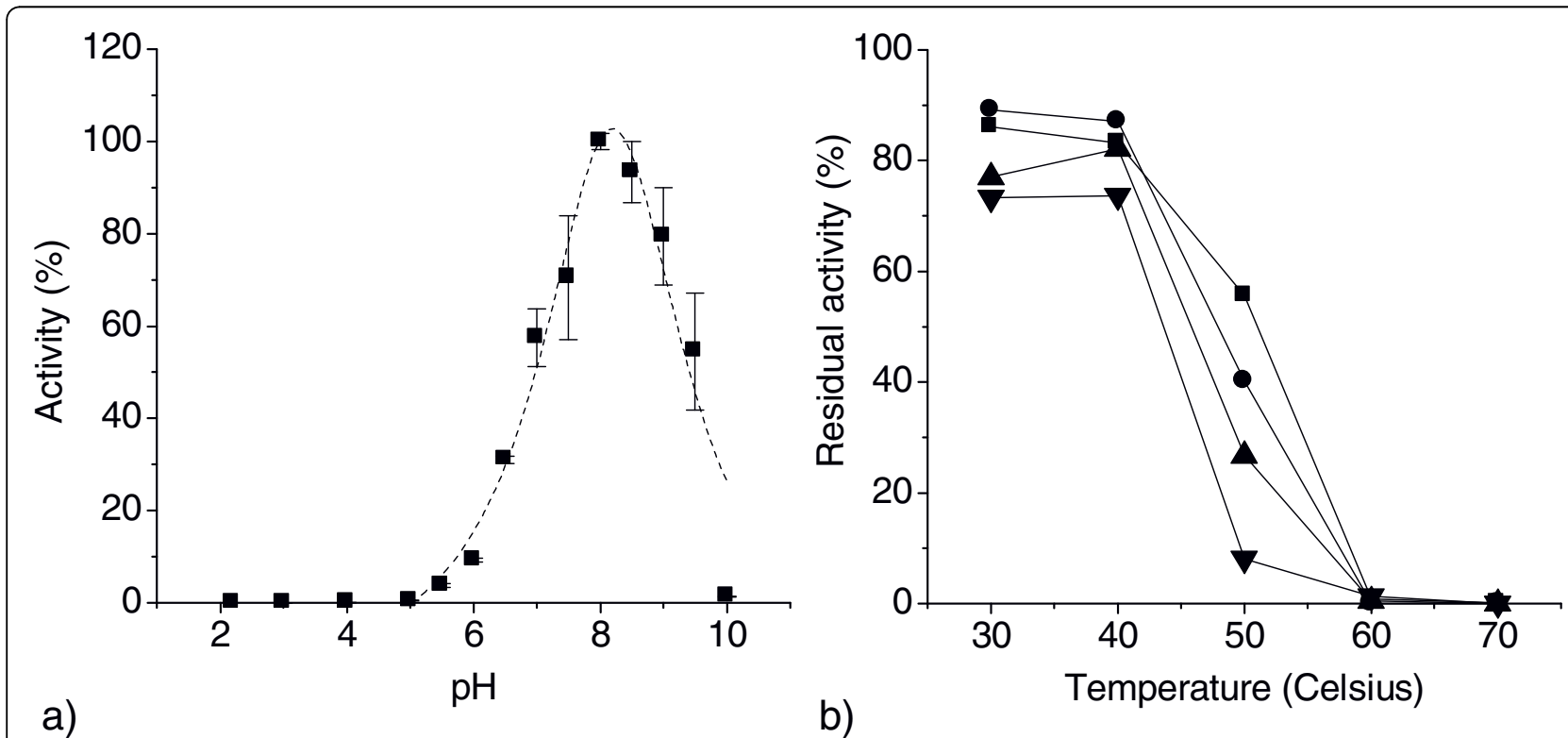

Figure $3 \mathrm{pH}$ optimum and temperature stability of AoSOX1. Sulfhydryl oxidase activity was measured with the HVA-peroxidase coupled assay on glutathione $5 \mathrm{mM}$ at room temperature to determine (a) the $\mathrm{pH}$ optimum and (b) temperature stability after 30 minutes (filled square), 1 hour (filled circle), 2 hours (filled triangle) and 24 hours (filled inverted triangle) of incubation at different temperatures. 


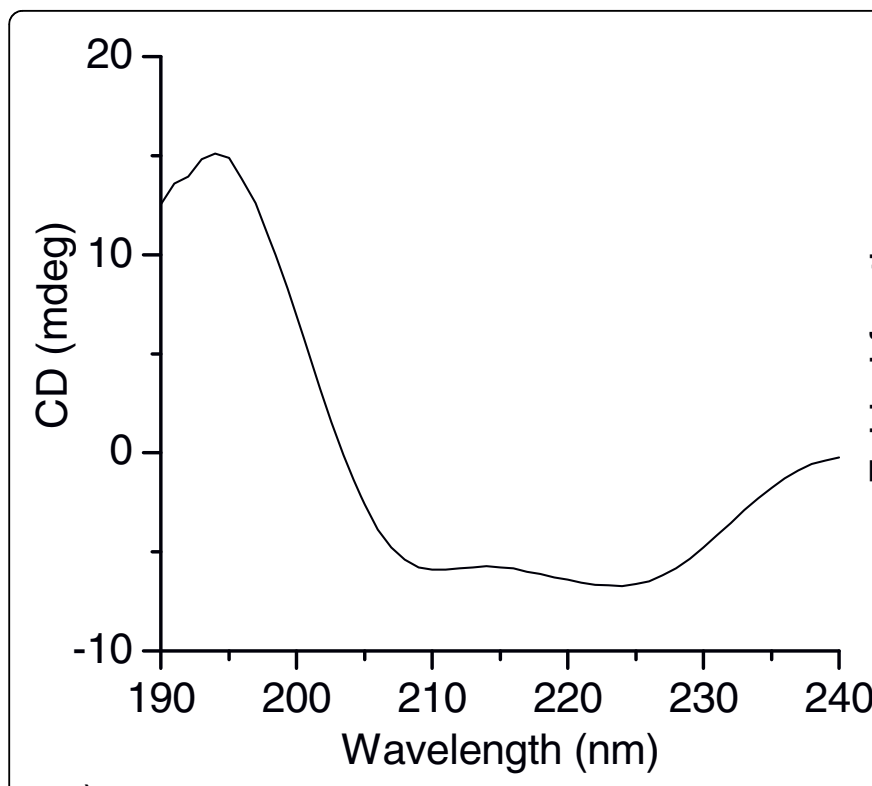

a)

Figure 5 Spectroscopic analysis of AoSOX1 by circular dichroism. (a) Spectra in the far-ultraviolet region of AoSOX 1 in native form at $25^{\circ} \mathrm{C}$ and (b) thermal denaturation of AoSOX1 assayed by circular dichroism.

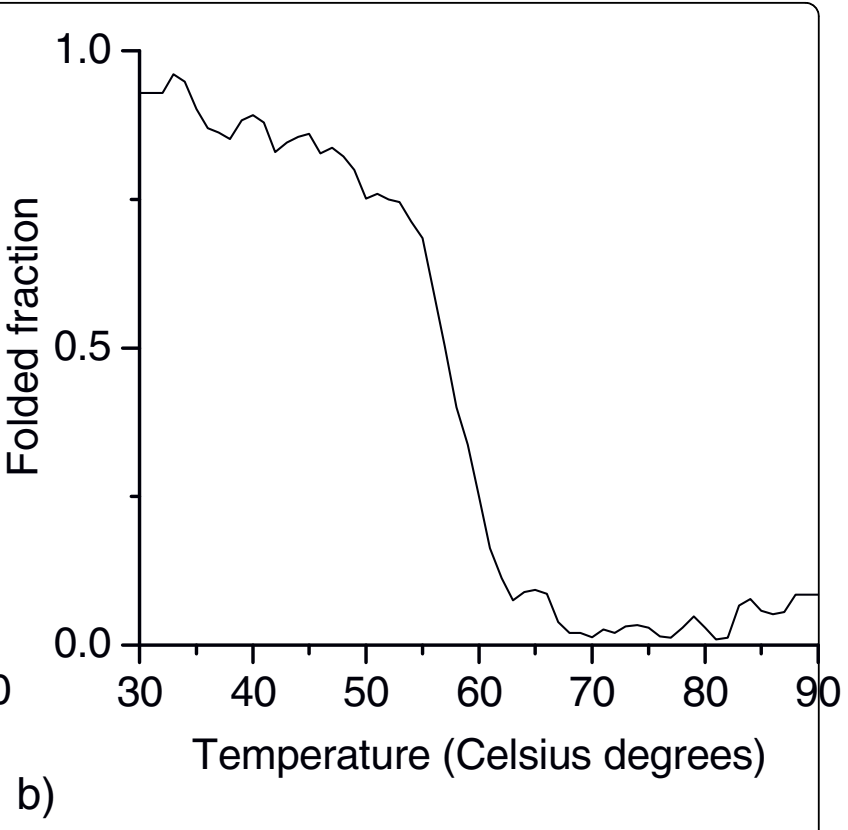

b) equilibrium unfolding measurements (Figure 6). The exposure of the tryptophan side chains to a more polar environment, as a result of protein unfolding was reflected in a red shift of the fluorescence peak from 334 to $355 \mathrm{~nm}$ and was completed at a $3.5 \mathrm{M}$ denaturant concentration. Tryptophan and FAD fluorescence had both a marked increase between 2 and $2.5 \mathrm{M}$ denaturant concentration that can be due to the presence of three of the six tryptophan residues of AoSOX1 (W154, W160 and W163) in close proximity of the catalytically active di-cysteine pair and thus possibly to the

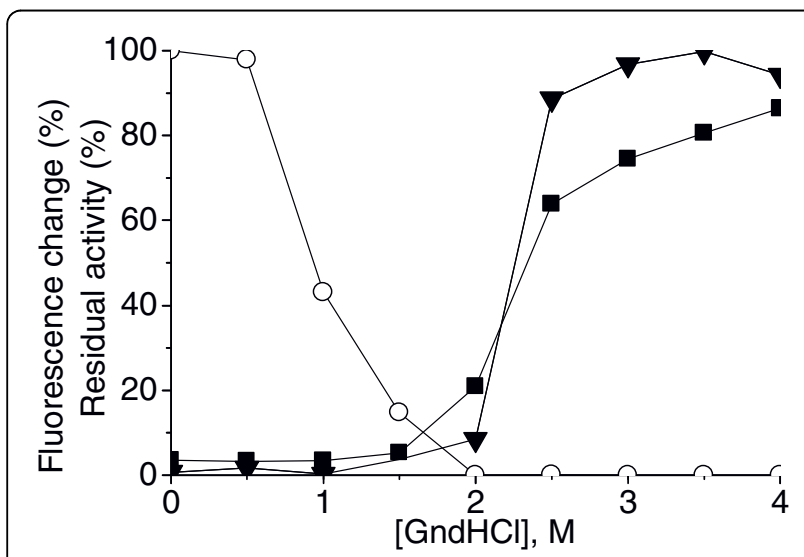

Figure 6 Equilibrium denaturation curves of AoSOX1. Intrinsic tryptophan fluorescence (triangle), flavin fluorescence (filled square) and residual activity (empty circle) of AoSOX1 were monitored at increasing guanidinium hydrochloride $(\mathrm{GndHCl})$ concentration. isoalloxazinic ring of the FAD cofactor. A drastic loss of activity was observed at a denaturant concentration above $1 \mathrm{M}$.

\section{Conclusions}

In the present study the sequences of potentially secreted fungal sulfhydryl oxidases were analyzed and a novel representative enzyme, AoSOX1 from A. oryzae, was produced in $T$. reesei in an active form. The characteristics of the enzymes are typical for sulfhydryl oxidases: the enzyme is capable of oxidizing small molecular compounds like glutathione, DTT and cysteine and the enzyme has a non-covalently bound FAD as a cofactor. Further testing of the activity on thiol groups within larger peptides and on protein level will be needed to assess the application potential of this enzyme.

\section{Methods}

All chemicals were purchased from Sigma-Aldrich but D-cysteine hydrochloride monohydrate that was purchased from Fluka. All columns for protein purification were purchased from GE Healthcare and all the purification steps were carried out with an Äkta purifier system (Amersham Biosciences).

\section{SOX sequence analysis}

An in-house database containing 28 public fungal genomes [19] was analysed by InterProScan program [20] for the presence of a pyridine nucleotide disulphide 
oxidoreductases domain of class II and, in particular, FAD-dependent ones (IPR000103 and IPR013027 respectively). Presence of a target peptide for the secretory pathway was considered and determined with TargetP [21]. Multiple sequence alignments were produced with the software ClustalW [22] and pairwise alignments at EMBL-EBI (http://www.ebi.ac.uk/Tools/emboss/align/ index.html).

\section{Isolation and expression of AoSOX1 gene in Trichoderma reesei}

The gene coding for AoSOX1 was amplified by PCR from the genomic DNA of $A$. oryzae, strain VTT-D88348, and with Dynazyme EXT polymerase (Finnzymes, Helsinki, Finland). The PCR program included an initial denaturation step of $3 \mathrm{~min}$ at $98^{\circ} \mathrm{C}$, followed by 25 cycles of $30 \mathrm{~s}$ at $98^{\circ} \mathrm{C}, 30 \mathrm{~s}$ at $60^{\circ} \mathrm{C}$ and $45 \mathrm{~s}$ at $72^{\circ} \mathrm{C}$. This was followed by a final elongation step of $10 \mathrm{~min}$ at $72^{\circ} \mathrm{C}$. Cloning was done with the Gateway technology (Invitrogen) and the primers were designed to incorporate $a t t B$ sites in the PCR product to allow the insertion, via $\mathrm{BP}$ reaction, into the $\mathrm{pDONR} 221$ cloning vector $\left(5^{\prime}\right.$ GGGGACAAGT TTGTACAAAA AAGCAGGCTA TCATGGCTCC TAAGTCTCTT TTCTAC3', 3'GGGG ACCACT TTGTACAAGA AAGCTGGGTT CAGTGG TGGT GGTGGTGGTG CAGGCCTCTA GACCGATTAT A5'). Primers were also designed to introduce a C-terminal tag of six histidines to the gene product. The subsequent LR recombination reaction transferred AoSOX1 gene into the pMS186 expression vector producing the plasmids pGF008. The pMS186 contained the Gateway reading frame cassette $\mathrm{C}$ between the $c b h 1$ (cellobiohydrolase 1) promoter and terminator, and a hygromycin resistance cassette. The recombinant plasmid was transformed as described [23] into a variant of the T. reesei strain VTT-D-00775 [24]. Transformants were streaked twice consecutively on plates containing hygromycin B $(125 \mu \mathrm{g} / \mathrm{ml})$ and then screened with PCR for the presence of the expression construct. Positive transformants were purified to single spore cultures and grown in shake flasks for 9 days at $28^{\circ} \mathrm{C}$ in $50 \mathrm{ml}$ of Trichoderma minimal medium supplemented with $4 \%$ lactose, $2 \%$ spent grains and $100 \mathrm{mM}$ piperazine- $\mathrm{N} \mathrm{N}^{\prime}$ Bis (3-propanesulfonic acid) pH 5.5. SOX activity was assayed after 5, 7 and 9 days on reduced L-glutathione. The transformant giving the highest activity was grown in $250 \mathrm{ml}$ culture medium in a $2 \mathrm{~L}$ flask at $28^{\circ} \mathrm{C}$ for 5 days for routine protein production and subsequent characterisation.

\section{Activity measurements}

\section{Oxygen consumption assay}

The reaction was initiated by the addition of the enzyme to $1.8 \mathrm{ml}$ of buffered substrate solution in a fully filled-in vial and the oxidation rate $\left(\mathrm{nmol} \mathrm{l}^{-1} \mathrm{~s}^{-1}\right)$ was calculated from the linear part of the oxygen consumption curve. OXY-10 mini-multi-channel oxygen meter (PreSens Precision Sensing Gmbh, Germany) was used in the measurements.

\section{HVA-peroxidase coupled assay}

SOX activity was generally measured in a 96 well microtiter plate with a coupled assay modified from Raje [25] where $10 \mu \mathrm{l}$ of enzyme solution was added to $95 \mu \mathrm{l}$ of a 1:1 mixture of $1.4 \mu \mathrm{M}$ peroxidase type II and $1 \mathrm{mM}$ homovanillic acid (HVA); the reaction was started adding $55 \mu \mathrm{l}$ of substrate, e.g. $5 \mathrm{mM}$ reduced L-glutathione. Reagents were dissolved in $50 \mathrm{mM}$ potassium phosphate buffer, $0.3 \mathrm{mM}$ EDTA pH 7.5. The production of the fluorescent HVA dimer was followed at excitation wavelength $320 \mathrm{~nm}$ and emission wavelength $420 \mathrm{~nm}$. The activity as variation of fluorescence was calculated in arbitrary units (AU) per minute. Activity measurements were performed in a black 96-well microtiter plate and fluorescence was measured using a Varioskan spectral scanning multimode reader (Thermo Electron Co., Vantaa, Finland).

The conversion of activity values in $\mathrm{AU}$ to kat $\mathrm{ml}^{-1}$ was possible with a calibration curve (Figure 7). Reaction mixtures containing different amounts of monomeric HVA were monitored to completion and the final fluorescence value was plotted against the number of moles of HVA dimer theoretically produced, and stoichiometrically equivalent to the molecules of oxygen consumed. In example, a standard reaction mixture for activity measurement contained $0.3 \mathrm{mM}$ monomeric

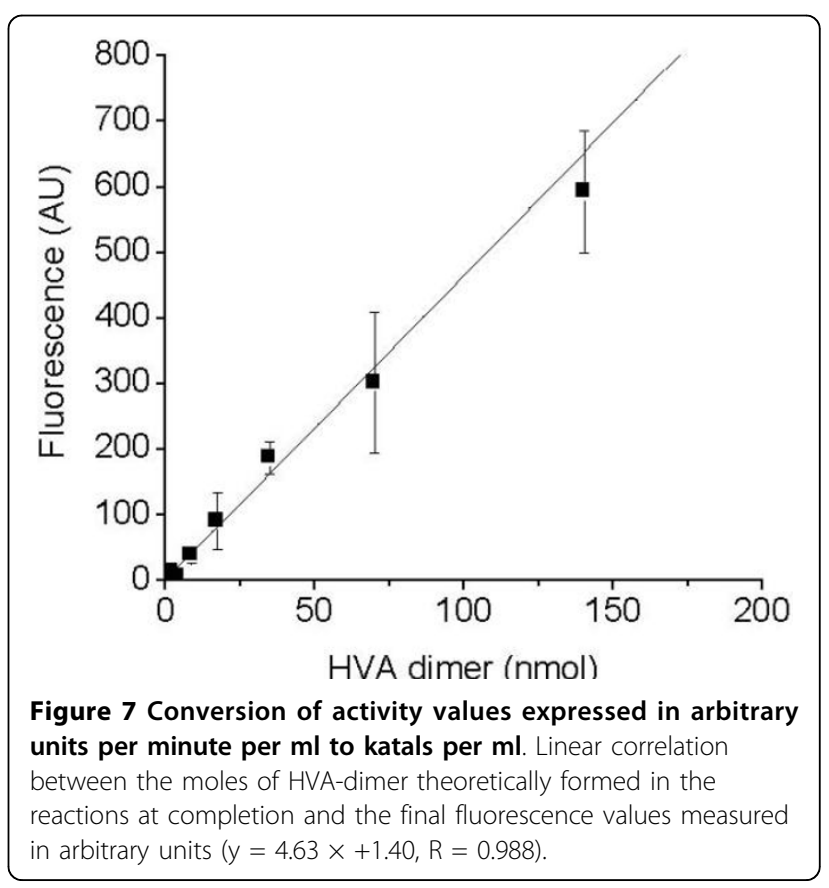


HVA that during a standard activity assay could lead to the formation of a maximum of 24 nmoles in dimeric form, in a $160 \mu \mathrm{l}$ reaction volume. Three identical reaction mixtures were measured, and a conversion factor of 8.6 was obtained for converting the activity values AU $\mathrm{ml}^{-1} \mathrm{~min}^{-1}$ to nkat $\mathrm{ml}^{-1}$.

The final reaction mixtures were analysed similarly to [26] by HPLC (Hypersil BDS C-18 $5 \mu \mathrm{m}, 4.6 \times 150 \mathrm{~mm}$, Agilent, operated by a Waters $600 \mathrm{E}$ system controller). The retention time of residual HVA monomer in the reaction mixtures was determined by comparison to solutions of HVA and measuring the absorbance of the eluate at $280 \mathrm{~nm}$ (Waters 2996 Photodiode Array detector). The HVA dimer elution was monitored by measuring the fluorescence $\left(\lambda_{\text {ex }}=315 \mathrm{~nm}, \lambda_{\text {em }}=425 \mathrm{~nm}\right.$, Waters474 Scanning Fluorescence detector). The retention time of HVA in the monomeric form was $2.6 \mathrm{~min}-$ utes while it was 3.2 minutes for the dimeric form. Dimerization of HVA in the reaction mixtures was confirmed by the appearance of a major fluorescent peak corresponding to dimeric HVA in all reactions.

\section{Assay with Ellman's reagent}

Ellman's reagent, 5,5'-dithiobis(2-nitrobenzoic acid) (DTNB), was used in a qualitative assay for the detection of SOX activity in the fractions of the purification. The enzyme sample of $10 \mu \mathrm{l}$ was incubated with $30 \mu \mathrm{l}$ of $13.3 \mathrm{mM}$ glutathione for 1 hour and the reaction was stopped by adding $300 \mu \mathrm{l}$ of $0.1 \mathrm{mM}$ DTNB. Reagents were dissolved in PBS buffer $\left(75 \mathrm{mM} \mathrm{KH_{2 }} \mathrm{PO}_{4}, 68 \mathrm{mM}\right.$ $\mathrm{NaCl} \mathrm{pH} \mathrm{7.5).}$

\section{Protein purification}

Cell-free medium containing AoSOX1 (220 ml) was concentrated by ultrafiltration (Amicon) using a 10000 cut-off ultrafiltration membrane (Millipore, Espoo, Finland), and was exchanged by dialysis into $20 \mathrm{mM}$ Tris$\mathrm{HCl} \mathrm{pH} 7$ at $4^{\circ} \mathrm{C}$. Proteins were separated by anion exchange chromatography (column Hitrap DEAE FF) and eluted in 25 column volumes with a linear 0-300 $\mathrm{mM} \mathrm{NaCl}$ gradient. Fractions showing a significant activity were then pooled and AoSOX1 was further purified by size-exclusion chromatography (column Superdex $75 \mathrm{HR}$ 10/30, $0.5 \mathrm{ml} \mathrm{min}^{-1}$ flow) in $20 \mathrm{mM}$ Tris$\mathrm{HCl} \mathrm{pH} \mathrm{7,} 150 \mathrm{mM} \mathrm{NaCl}$.

$\mathrm{N}$-glycans of AoSOX1 were removed from the denatured protein with a PNGase F treatment according to the manufacturer's instructions (Calbiochem, Merck KGaA Darmstadt, Germany) and the result was visualized by SDS PAGE. The protein content was determined with the Bio-Rad DC (Bio-Rad, Richmond, CA, USA) protein assay kit. The purity was estimated to be higher than $95 \%$ by electrophoresis in a $12 \%$ SDS PAGE [27], using a Pre-stained SDS PAGE Standard (GE Healthcare, Uppsala, Sweden) and Coomassie Brilliant
Blue (Pharmacia Biotech, St. Albans, UK). Purified AoSOX1 protein concentration was determined using the extinction coefficient $12160 \mathrm{M}^{-1} \mathrm{~cm}^{-1}$ at $450 \mathrm{~nm}$ of the cofactor that was experimentally determined based on the amount of released FAD after SDS (0.2\%) and heat treatment (10-30 minutes at $95^{\circ} \mathrm{C}$ in the dark).

\section{Spectroscopic methods (circular dichroism, UV/visible and fluorescence spectroscopy)}

Absorption spectra were measured in $20 \mathrm{mM}$ Tris- $\mathrm{HCl}$ pH 7.0 at $25^{\circ} \mathrm{C}$ using a Cary Varian 100 Bio UV-Vis spectrophotometer. Circular dichroism (CD) spectra were recorded on a JASCO model J-720 CD spectrometer equipped with a Peltier PTC-38WI thermally controlled cuvette holder. Far-UV CD measurements (190-240 $\mathrm{nm}$ ) were performed with $2.5 \mu \mathrm{M}$ purified enzyme in $0.1 \mathrm{M}$ sodium phosphate buffer $\mathrm{pH} 7$ at $25^{\circ} \mathrm{C}$, using a $1 \mathrm{~mm}$ cell and bandwidth of $1 \mathrm{~nm}$. Spectra were accumulated four times and the values were corrected for buffer contributions. Thermally induced denaturation was followed as the change in a signal at $222 \mathrm{~nm}$ in a $30-90^{\circ} \mathrm{C}$ temperature range with a $2^{\circ} \mathrm{C}$ $\min ^{-1}$ heating rate. Fluorescence spectra were recorded on a Varian Cary Eclipse Fluorescence Spectrophotometer using a $50 \mu \mathrm{M}$ AoSOX1 solution in $20 \mathrm{mM}$ Tris $\mathrm{pH} 7$ at $20^{\circ} \mathrm{C}$. Protein unfolding was done by the addition of amounts of a $6 \mathrm{M}$ guanidinium hydrochloride solution to get a 0 to $5.5 \mathrm{M}$ denaturant concentration in the sample. An incubation time of 15 minutes was allowed before spectrum recording. Excitation wavelength of $290 \mathrm{~nm}$ for the tryptophan residues and $450 \mathrm{~nm}$ for the flavin cofactor were used, and the fluorescence was recorded in a 300-450 nm and 450-600 nm wavelength intervals, respectively.

\section{Peptide fingerprinting and molecular weight determination}

Bands in the gel (SDS PAGE, 12\% acrylamide) were excised and the protein was treated essentially as described by Rosenfeld [28] for peptide mass fingerprinting experiments. The molecular weight of AoSOX1 was determined by MALDI TOF-MS after the enzyme was transferred to distilled water, sinapinic acid was used as matrix in the mass spectrometry. MALDI TOFMS experiments were carried out in a TOF mass spectrometer Autoflex II (Bruker Daltonik, Bremen, Germany) equipped with laser (160 pulse width, $50 \mathrm{~Hz}$ repetition rate) as described [29].

\section{Determination of $\mathrm{pH}$ optimum and stability measurements}

The enzymatic activity was measured with the HVAperoxidase coupled assay; to reduce the influence of $\mathrm{pH}$ on the substrate and the reagents of the assay, all 
solutions and the enzyme were used in a 10-fold higher concentration in assay buffer and diluted to the final concentration in buffer at the proper $\mathrm{pH}$ directly in the well of the microtiter plate, e.g. McIlvaine buffer $(\mathrm{pH}$ 2.2-8.0), $20 \mathrm{mM}$ Tris/ $\mathrm{HCl}(\mathrm{pH} 8.5)$ and $100 \mathrm{mM}$ CAPSO (pH 8.5-10). AoSOX1 pH stability was determined by incubating the enzyme in McIlvaine buffer (pH 2.2-8.0) and $20 \mathrm{mM}$ Tris/ $\mathrm{HCl}(\mathrm{pH} 8.5)$ and measuring the residual activity after 1 and 24 hours. Temperature stability was assayed incubating aliquots of enzyme solution at $30,40,50,60$ and $70^{\circ} \mathrm{C}$ and measuring the activity in withdrawn samples after 1 and 24 hours with the HVA-peroxidase coupled assay.

\section{Inhibition studies}

Sulfhydryl oxidase activity was measured on glutathione ( $5 \mathrm{mM}$ ) by oxygen consumption assay at room temperature in the presence of inhibitors (EDTA, KI, NaCl, $\mathrm{MgSO}_{4}, \mathrm{MnSO}_{4}, \mathrm{Na}_{2} \mathrm{SO}_{4}, \mathrm{ZnSO}_{4}$, SDS and urea) at 1 and $10 \mathrm{mM}$ concentrations in $50 \mathrm{mM}$ potassium phosphate buffer $\mathrm{pH}$ 7.5.

\section{Acknowledgements}

This work was supported by the Marie Curie EU-project PRO-ENZ (MEST-CT2005-020924) and by the Finnish Cultural Foundation. We are grateful to Harry Boer and Evanthia Monogioudi for the assistance in mass spectrometry and CD spectroscopy. The technical assistance of Mikko Arvas is acknowledged. We are thankful to Hanna Kuusinen, Outi Nivala and Outi Liehunen for the skilful support.

\section{Authors' contributions}

GF carried out the genome mining study, the experimental work and drafted the manuscript. MS and KK participated in the design and conceived of the study and helped to draft the manuscript. JB participated in the design and coordination of the study. All authors read and approved the final manuscript.

Received: 3 February 2010 Accepted: 20 August 2010

Published: 20 August 2010

\section{References}

1. Mandels GR: Properties and surface location of a sulfhydryl oxidizing enzyme in fungus spores. J Bacteriol 1956, 72(2):230-234.

2. Neufeld HA, Green LF, Latterell FM, Weintraub RL: Thioxidase, a new sulfhydryl-oxidizing enzyme from Piricularia oryzae and Polyporus versicolor. J Biol Chem 1958, 232(2):1093-1099.

3. Kusakabe $\mathrm{H}$, Kuninaka A, Yoshino H: Purification and Properties of a New Enzyme, Glutathione Oxidase from Penicillium sp. K-6-5. Agricultural and Biological Chemistry 1982, 46(8):2057-2067.

4. de la Motte RS, Wagner FW: Aspergillus niger sulfhydryl oxidase. Biochemistry 1987, 26(23):7363-7371.

5. Vignaud C, Kaid N, Rakotozafy L, Davidou S, Nicolas J: Partial Purification and Characterization of Sulfhydryl Oxidase from Aspergillus niger. J Food Sci 2002, 67(6):2016-2022.

6. Thorpe C, Hoober KL, Raje S, Glynn NM, Burnside J, Turi GK, Coppock DL: Sulfhydryl oxidases: emerging catalysts of protein disulfide bond formation in eukaryotes. Arch Biochem Biophys 2002, 405(1):1-12.

7. Swaisgood HE, Abraham P: Oxygen Activation by Sulfhydryl Oxidase and the Enzyme's Interaction with Peroxidase. J Dairy Sci 1980, 63:1205-1210.

8. Yamada H: Localization in skin, activation and reaction mechanisms of skin sulfhydryl oxidase. Nippon Hifuka Gakkai Zasshi 1989, 99:861-869.

9. Vemulapalli V, Miller KA, Hoseney RC: Glucose Oxidase in Breadmaking Systems. Cereal Chem 1998, 75(4):439-442.
10. Kleiger G, Eisenberg D: GXXXG and GXXXA motifs stabilize FAD and NAD (P)-binding Rossmann folds through $\mathrm{C}(\mathrm{alpha})-\mathrm{H}$... O hydrogen bonds and van der waals interactions. J Mol Biol 2002, 323(1):69-76.

11. Fass D: The Erv family of sulfhydryl oxidases. Biochim Biophys Acta 2008, 1783(4):557-566.

12. Vallon $\mathrm{O}$ : New sequence motifs in flavoproteins: Evidence for common ancestry and tools to predict structure. Proteins: Structure, Function, and Genetics 2000, 38(1):95-114.

13. Chivers PT, Prehoda KE, Raines RT: The CXXC motif: a rheostat in the active site. Biochemistry 1997, 36(14):4061-4066.

14. Quan S, Schneider I, Pan J, Von Hacht A, Bardwell JC: The CXXC motif is more than a redox rheostat. J Biol Chem 2007, 282(39):28823-28833.

15. Eggink $G$, Engel $H$, Vriend $G$, Terpstra P, Witholt B: Rubredoxin reductase of Pseudomonas oleovorans. Structural relationship to other flavoprotein oxidoreductases based on one NAD and two FAD fingerprints. J Mol Biol 1990, 212(1):135-142.

16. Letunic I, Copley RR, Pils B, Pinkert S, Schultz J, Bork P: SMART 5: domains in the context of genomes and networks. Nucleic Acids Res 2006, 34 Database: D257-60.

17. Hoober KL: Homology between Egg White Sulfhydryl Oxidase and Quiescin Q6 Defines a New Class of Flavin-linked Sulfhydryl Oxidases. J Biol Chem 1999, 274(45):31759.

18. Wang C, Wesener SR, Zhang H, Cheng YQ: An FAD-dependent pyridine nucleotide-disulfide oxidoreductase is involved in disulfide bond formation in FK228 anticancer depsipeptide. Chem Biol 2009, 16(6):585-593.

19. Arvas M, Kivioja T, Mitchell A, Saloheimo M, Ussery D, Penttila M, Oliver S: Comparison of protein coding gene contents of the fungal phyla Pezizomycotina and Saccharomycotina. BMC Genomics 2007, 8:325.

20. Quevillon E, Silventoinen V, Pillai S, Harte N, Mulder N, Apweiler R, Lopez R: InterProScan: protein domains identifier. Nucleic Acids Res 2005, 33:116-120.

21. Emanuelsson $\mathrm{O}$, Brunak S, von Heijne $\mathrm{G}$, Nielsen $\mathrm{H}$ : Locating proteins in the cell using TargetP, SignalP and related tools. Nat Protoc 2007, 2(4):953-971.

22. Larkin MA, Blackshields G, Brown NP, Chenna R, McGettigan PA, McWilliam H, Valentin F, Wallace IM, Wilm A, Lopez R, Thompson JD, Gibson TJ, Higgins DG: Clustal W and Clustal X version 2.0. Bioinformatics 2007, 23(21):2947-2948

23. Penttila $M$, Nevalainen $H$, Ratto $M$, Salminen $E$, Knowles J: A versatile transformation system for the cellulolytic filamentous fungus Trichoderma reesei. Gene 1987, 61(2):155-164

24. Kontkanen H, Westerholm-Parvinen A, Saloheimo M, Bailey M, Rättö M, Mattila M, Mohsina M, Kalkkinen N, Nakari-Setälä T, Buchert J: Novel Coprinopsis cinerea polyesterase that hydrolyzes cutin and suberin. Appl Environ Microbiol 75(7):2148-2157.

25. Raje S, Glynn NM, Thorpe C: A continuous fluorescence assay for sulfhydryl oxidase. Anal Biochem 2002, 307(2):266-272.

26. Foppoli C, Coccia R, Blarzino C, Rosei MA: Formation of homovanillic acid dimer by enzymatic or Fenton system - catalyzed oxidation. IJBCB 2000, 32:657-663.

27. Laemmli UK: Cleavage of structural proteins during the assembly of the head of bacteriophage T4. Nature 1970, 227(5259):680-685.

28. Rosenfeld J, Capdevielle J, Guillemot JC, Ferrara P: In-gel digestion of proteins for internal sequence analysis after one- or two-dimensional gel electrophoresis. Anal Biochem 1992, 203(1):173-179.

29. Selinheimo E, Saloheimo M, Ahola E, Westerholm-Parvinen A, Kalkkinen N, Buchert J, Kruus K: Production and characterization of a secreted, C-terminally processed tyrosinase from the filamentous fungus Trichoderma reesei. FEBS J 2006, 273(18):4322-4335.

doi:10.1186/1471-2091-11-31

Cite this article as: Faccio et al:: Secreted fungal sulfhydryl oxidases: sequence analysis and characterisation of a representative flavindependent enzyme from Aspergillus oryzae. BMC Biochemistry 2010 11:31. 